Mathematical Modelling and Analysis

Volume 21 Number 2, March 2016, 199-219

http://dx.doi.org/10.3846/13926292.2016.1146925

(c) Vilnius Gediminas Technical University, 2016
Publisher: Taylor\&Francis and VGTU

http://www.tandfonline.com/TMMA

ISSN: $1392-6292$

eISSN: 1648-3510

\title{
Mixed Pseudospectral Method for Heat Transfer
}

\section{Tian-Jun Wang ${ }^{a}$ and Tao Sun ${ }^{b}$}

${ }^{a}$ School of Mathematics and Statistics, Henan University of Science and Technology

Kaiyuan Road Luolong District No. 263, 471003 Luoyang, China

${ }^{b}$ School of Statistics and Mathematics, Shanghai Finance University

Shangchuan Road Pudong District No. 995, 201209 Shanghai, China

E-mail(corresp.): wangtianjun64@163.com

E-mail: stao_2004@126.com

Received September 12, 2015; revised January 22, 2016; published online March 15, 2016

\begin{abstract}
In this paper, we propose a mixed generalized Laguerre-Legendre pseudospectral method for non-isotropic heat transfer with inhomogeneous boundary conditions on an infinite strip. Some properties about the mixed generalized LaguerreLegendre approximation are established. By reformulating the equation with suitable functional transform defined on an infinite strip, a mixed Laguerre-Legendre pseudospectral scheme is constructed. Its convergence is proved. Numerical results are presented to demonstrate the efficiency of this new approach and to validate our theoretical analysis.
\end{abstract}

Keywords: mixed Laguerre-Legendre approximation, pseudospectral method for heat transfer, inhomogeneous Dirichlet boundary condition, infinite strip.

AMS Subject Classification: 65M70; 35K05; 35K99; 35R15; 41A30.

\section{Introduction}

Spectral methods have been successfully used for numerical simulations of various problems in science and engineering, see [1,2,3,7,20]. Typically, Fourier spectral methods are used for periodic problems, and the Legendre and Chebyshev spectral methods are used for problems defined on bounded domains. For problems on unbounded domains, some authors also studied Hermite and Laguerre approximations, see $[4,5,6,8,9,12,13,14,17,18,19,21,23,26,27,28]$. Recently, Guo and Zhang [16], Wang [25] considered the spectral method for differential equations of degenerate type, by using the following scaled generalized Laguerre functions

$$
\tilde{\mathcal{L}}_{l}^{(\alpha, \beta)}(x)=e^{-\frac{1}{2} \beta x} \mathcal{L}_{l}^{(\alpha, \beta)}(x)=\frac{1}{l !} x^{-\alpha} e^{\frac{1}{2} \beta x} \partial_{x}^{l}\left(x^{l+\alpha} e^{-\beta x}\right), \quad \alpha>-1, \beta>0,
$$


which are mutually orthogonal with the weight function $x^{\alpha}$. By adjusting the parameter $\alpha$ properly, we can derive simple alternative Galerkin variational formulations, which could be approximated directly and implemented easily. Moreover, the adjustable parameter $\beta$ offers great flexibility to match the asymptotic behaviors of exact solutions at infinity. It is noted that Shen [19] used such system with $\alpha=0$ and $\beta=1$ for solving one-dimensional differential equations.

In this paper, we consider the non-isotropic heat transfer in an infinite strip. A straightforward approach is to truncate the infinite strip into a sufficiently large subdomain and impose certain artificial boundary conditions. However, generally it is difficult to find an accurate and effective boundary condition. By using the fact that Laguerre functions are defined over infinite long intervals, the authors proposed a mixed generalized Laguerre-Legendre spectral method for solving this problem, see [25]. In practical computation, pseudospectral methods are more preferable since we only need to evaluate unknown functions at the interpolation nodes. In particular, it is much easier to deal with nonlinear heat transfer process.

The aim of this paper is to develop the mixed generalized Laguerre-Legendre pseudospectral method for non-isotropic heat transfer in an infinite strip, by using the Legendre interpolation in the direction of finite length, and the generalized Laguerre function interpolation in the infinite long direction. Rigorous error estimates on the Jacobi interpolation and generalized Laguerre interpolation were well established, see $[15,24]$. It is well known that the solutions of heat transfer process decay very fast as $x \rightarrow \infty$. Thus it is more appropriate to take the Laguerre functions $e^{\frac{1}{2} \beta x} \mathcal{L}_{l}^{(\alpha, \beta)}(x)$ as the basis functions for this type of problems so that the asymptotic behavior of the solutions can be better fitted. Moreover, the use of scaled generalized Laguerre functions lead to much simplified analysis, more precise error estimates. Wang [21] studied the generalized Laguerre spectral method for Fisher's equation on a semi-infinite interval, Wang [22] also investigated a mixed spectral method for heat transfer with Neumann boundary condition in an infinite strip by reformulating the equation with suitable functional transform. Follow a similar idea as in [21,22], we shall reformulate heat transfer with inhomogeneous Dirichlet boundary conditions to some alternative forms with homogeneous boundary conditions imposed on some parts of the boundary with suitable functional transform defined on an infinite strip. At the same time, the auxiliary function may also simulate the asymptotic behaviors of the solution of heat transfer. Then, we construct a mixed Laguerre-Legendre pseudospectral scheme for non-isotropic heat transfer in an infinite strip. We establish some basic results on this mixed LaguerreLegendre approximation, from which the convergence of proposed scheme follows. We also design an efficient algorithm for implementation and present some numerical results to validate the efficiency of this approach.

This paper is organized as follows. In Section 2, we recall some basic formulas for Laguerre approximation, Legendre approximation and the mixed Laguerre-Legendre interpolation approximation. Then we construct the mixed Laguerre-Legendre pseudospectral scheme for non-isotropic heat transfer, investigate two useful mixed orthogonal projections, which play important roles 
in the numerical analysis of spectral methods for various differential equations in an infinite strip. The convergence of proposed schemes are proved in Section 3. We present a numerical example to demonstrate the high accuracy of proposed algorithm in Section 4. The final section is for some concluding remarks.

\section{Mixed Laguerre-Legendre Interpolation}

In this section, we recall the basic results on the mixed Laguerre-Legendre interpolation.

\subsection{Laguerre Interpolation}

We first consider the one-dimensional Laguerre-Gauss-Radau interpolation. Let $\Lambda=\{x \mid 0<x<\infty\}$ and $\chi(x)$ be a certain weight function. For integer $r \geq 0$,

$$
H_{\chi}^{r}(\Lambda)=\left\{u \mid u \text { is measurable on } \Lambda \text { and }\|u\|_{r, \chi, \Lambda}<\infty\right\},
$$

equipped with the following inner product, semi-norm and norm

$$
\begin{aligned}
& (u, v)_{r, \chi, \Lambda}=\sum_{0 \leq k \leq r} \int_{\Lambda} \partial_{x}^{k} u(x) \partial_{x}^{k} v(x) \chi(x) d x \\
& |u|_{r, \chi, \Lambda}=\int_{\Lambda}\left(\partial_{x}^{r} u(x)\right)^{2} \chi(x) d x, \quad\|u\|_{r, \chi, \Lambda}=(u, u)_{r, \chi, \Lambda}^{\frac{1}{2}} .
\end{aligned}
$$

In particular, $H_{\chi}^{0}(\Lambda)=L_{\chi}^{2}(\Lambda)$, with the inner product $(u, v)_{\chi, \Lambda}$ and the norm $\|u\|_{\chi, \Lambda}$. We omit the subscript $\chi$ in the notations whenever $\chi(x) \equiv 1$.

Let $\omega_{\alpha, \beta}(x)=x^{\alpha} e^{-\beta x}, \alpha>-1, \beta>0$. Especially, $\omega_{\beta}(x)=\omega_{0, \beta}(x)=e^{-\beta x}$. The scaled Laguerre polynomial of degree $l$ is defined by

$$
\mathcal{L}_{l}^{(\beta)}(x)=\frac{1}{l !} e^{\beta x} \partial_{x}^{l}\left(x^{l} e^{-\beta x}\right) .
$$

To design proper pseudospectral method for heat transfer, we use the Laguerre functions as

$$
\tilde{\mathcal{L}}_{l}^{(\beta)}(x)=e^{-\frac{1}{2} \beta x} \mathcal{L}_{l}^{(\beta)}(x), \quad l=0,1,2, \cdots
$$

The set of $\tilde{\mathcal{L}}_{l}^{(\beta)}(x)$ is a complete $L^{2}(\Lambda)$-orthogonal system (cf. [16]).

For any positive integer $N, \mathcal{P}_{N}(\Lambda)$ stands for the set of all polynomials of degree at most $N$. Let $\xi_{R, N, \Lambda, j}^{(\beta)}, 0 \leq j \leq N$, be the zeros of polynomial $x \partial_{x} \mathcal{L}_{N+1}^{(\beta)}(x)$, which are arranged in ascending order. Denote by $\omega_{R, N, \Lambda, j}^{(\beta)}, 0 \leq$ $j \leq N$, the corresponding Christoffel numbers such that

$$
\int_{\Lambda} \phi(x) \omega_{\beta}(x) d x=\sum_{j=0}^{N} \phi\left(\xi_{R, N, \Lambda, j}^{(\beta)}\right) \omega_{R, N, \Lambda, j}^{(\beta)}, \quad \forall \phi \in \mathcal{P}_{2 N}(\Lambda) .
$$


Furthermore, let $\tilde{\xi}_{R, N, \Lambda, j}^{(\beta)}=\xi_{R, N, \Lambda, j}^{(\beta)}$ and $\tilde{\omega}_{R, N, \Lambda, j}^{(\beta)}=e^{\beta \xi_{R, N, \Lambda, j}^{(\beta)} \omega_{R, N, \Lambda, j}^{(\beta)}}$. We also introduce the following discrete inner product and norm,

$$
(u, v)_{N, \beta, \Lambda}=\sum_{j=0}^{N} u\left(\tilde{\xi}_{R, N, \Lambda, j}^{(\beta)}\right) v\left(\tilde{\xi}_{R, N, \Lambda, j}^{(\beta)}\right) \tilde{\omega}_{R, N, \Lambda, j}^{(\beta)}, \quad\|u\|_{N, \beta, \Lambda}=(u, u)_{N, \beta, \Lambda}^{\frac{1}{2}} .
$$

Next, we set $\mathcal{Q}_{N, \beta}(\Lambda)=\left\{e^{-\frac{1}{2} \beta x} \psi \mid \psi \in \mathcal{P}_{N}(\Lambda)\right\}$. Let $\bar{\Lambda}=\Lambda \cup\{x=0\}$. For any $u \in C(\bar{\Lambda})$, the Laguerre-Gauss-Radau interpolation $\tilde{\mathcal{I}}_{R, N, \beta, \Lambda} u \in \mathcal{Q}_{N, \beta}(\Lambda)$ is determined by

$$
\tilde{\mathcal{I}}_{R, N, \beta, \Lambda} u\left(\tilde{\xi}_{R, N, \Lambda, j}^{(\beta)}\right)=u\left(\tilde{\xi}_{R, N, \Lambda, j}^{(\beta)}\right), \quad 0 \leq j \leq N .
$$

In the forthcoming discussions, we denote by $c$ a generic positive constant which does not depend on $M, N, \beta$ and any function.

In order to obtain precise error estimates of pseudospectral method for the first boundary values problems, we introduce the following orthogonal projection. For this purpose, let

$$
{ }_{0} H^{1}(\Lambda)=\left\{u \mid u \in H^{1}(\Lambda) \text { and } u(0)=0\right\}, \quad{ }_{0} \mathcal{Q}_{N, \beta}(\Lambda)={ }_{0} H^{1}(\Lambda) \cap \mathcal{Q}_{N, \beta}(\Lambda) .
$$

The orthogonal projection: ${ }_{0} P_{N, \beta, \Lambda}^{1}:{ }_{0} H^{1}(\Lambda) \rightarrow{ }_{0} \mathcal{Q}_{N, \beta}(\Lambda)$ is given by

$$
\left(\partial_{x}\left({ }_{0} P_{N, \beta, \Lambda}^{1} u-u\right), \partial_{x} \phi\right)_{\Lambda}=0, \quad \forall \phi \in{ }_{0} \mathcal{Q}_{N, \beta}(\Lambda) .
$$

Following the same line as in the proof of Lemma 2.2 of [13], we have that, if $u \in{ }_{0} H^{1}(\Lambda), \partial_{x}^{r}\left(e^{\frac{1}{2} \beta x} u\right) \in L_{\omega_{r-1, \beta}}^{2}(\Lambda)$, integer $r \geq 1$ and $r \leq N+1$, then

$$
\left\|\partial_{x}^{s}\left({ }_{0} P_{N, \beta, \Lambda}^{1} u-u\right)\right\|_{\Lambda} \leq c \beta^{s-1}(\beta N)^{\frac{1-r}{2}}\left\|\partial_{x}^{r}\left(e^{\frac{1}{2} \beta x} u\right)\right\|_{\omega_{r-1, \beta}, \Lambda}, \quad s=0,1 .
$$

\subsection{Legendre-Gauss-Lobatto interpolation}

We now turn to the Legendre-Gauss-Lobatto interpolation on the interval $I=$ $\{y|| y \mid<1\}$. For integer $r \geq 0$, we define the space $H^{r}(I)$ and its norm $\|u\|_{r, I}$ as usual. In particular, $L^{2}(I)=H^{0}(I)$ with the inner product $(u, w)_{I}$ and the norm $\|u\|_{I}=\|u\|_{0, I}$.

The Legendre polynomial of degree $l$ is defined by

$$
L_{l}(y)=\frac{(-1)^{l}}{2^{l} l !} \partial_{y}^{l}\left(1-y^{2}\right)^{l} .
$$

The set of Legendre polynomials is a complete $L^{2}(I)$-orthogonal system.

Now, let $M$ be any positive integer. $\mathcal{P}_{M}(I)$ stands for the set of all polynomials of degree at most $M$. Let $\zeta_{L, M, I, k}$ be the roots of polynomial (1 $\left.y^{2}\right) \partial_{y} L_{M}(y), 0 \leq k \leq M$, which are arranged in ascending order. The corresponding Christoffel numbers are denoted by $\rho_{L, M, I, k}, 0 \leq k \leq M$. We also introduce the discrete inner product and norm as

$$
(u, v)_{M, I}=\sum_{k=0}^{M} u\left(\zeta_{L, M, I, k}\right) v\left(\zeta_{L, M, I, k}\right) \rho_{L, M, I, k}, \quad\|u\|_{M, I}=(u, u)_{M, I}^{\frac{1}{2}} .
$$


Let $\bar{I}=I \cup\{y= \pm 1\}$. For any $u \in C(\bar{I})$, the Legendre-Gauss-Lobatto interpolation $\mathcal{I}_{L, M, I} u \in \mathcal{P}_{M}(I)$ is determined by

$$
\mathcal{I}_{L, M, I} u\left(\zeta_{L, M, I, k}\right)=u\left(\zeta_{L, M, I, k}\right), \quad 0 \leq k \leq M
$$

Let $H_{0}^{1}(I)=\left\{u \in H^{1}(I)\right.$ and $\left.u( \pm 1)=0\right\}$ and $\mathcal{P}_{M}^{0}(I)=H_{0}^{1}(I) \cap \mathcal{P}_{M}(I)$. The orthogonal projection $P_{M, I}^{1,0}: H_{0}^{1}(I) \rightarrow \mathcal{P}_{M}^{0}(I)$ is defined by

$$
\left(\partial_{y}\left(P_{M, I}^{1,0} u-u\right), \partial_{y} \phi\right)_{I}=0, \quad \forall \phi \in \mathcal{P}_{M}^{0}(I)
$$

According to Theorem 3.4 of [10], we know that if $u \in H_{0}^{1}(I),\left(1-y^{2}\right)^{\frac{q-1}{2}} \partial_{y}^{q} u \in$ $L^{2}(I)$ and integer $q \geq 1$ and $q \leq M+1$, then

$$
\left\|\partial_{y}^{s}\left(P_{M, I}^{1,0} u-u\right)\right\|_{I} \leq c M^{s-q}\left\|\left(1-y^{2}\right)^{\frac{q-1}{2}} \partial_{y}^{q} u\right\|_{I}, \quad s=0,1 .
$$

\subsection{Mixed Laguerre-Legendre Interpolation}

We now consider the mixed Laguerre-Legendre interpolation approximation on the domain $\Omega=\Lambda \times I$. Let $\chi(x, y)$ be a certain weight function. We define the weighted space $L_{\chi}^{2}(\Omega)$ in the usual way, with the following inner product and norm,

$$
(u, w)_{\chi, \Omega}=\iint_{\Omega} u(x, y) w(x, y) \chi(x, y) d x d y, \quad\|u\|_{\chi, \Omega}=(u, u)_{\chi, \Omega}^{\frac{1}{2}} .
$$

We omit the subscript $\chi$ in the notations whenever $\chi(x, y) \equiv 1$.

Let $V_{M, N, \beta}(\Omega)=\mathcal{P}_{M}(I) \otimes \mathcal{Q}_{N, \beta}(\Lambda)$. The meanings of $\tilde{\xi}_{R, N, \Lambda, j}^{(\beta)}, \tilde{\omega}_{R, N, \Lambda, j}^{(\beta)}$, $\zeta_{L, M, I, k}$ and $\rho_{L, M, I, k}$ are the same as before. The discrete inner product and norm are given by

$$
\begin{aligned}
& (u, v)_{M, N, \beta, \Omega}=\sum_{k=0}^{M} \sum_{j=0}^{N} u\left(\zeta_{L, M, I, k}, \tilde{\xi}_{R, N, \Lambda, j}^{(\beta)}\right) v\left(\zeta_{L, M, I, k}, \tilde{\xi}_{R, N, \Lambda, j}^{(\beta)}\right) \rho_{L, M, I, k} \tilde{\omega}_{R, N, \Lambda, j}^{(\beta)}, \\
& \|u\|_{M, N, \beta, \Omega}=(u, u)_{M, N, \beta, \Omega}^{\frac{1}{2}} .
\end{aligned}
$$

We have (cf. [24])

$$
\begin{aligned}
& (\phi, \psi)_{M, N, \beta, \Omega}=(\phi, \psi)_{\Omega}, \quad \forall \phi, \psi \in V_{M-1, N, \beta}(\Omega), \\
& \|\phi\|_{\Omega} \leq\|\phi\|_{M, N, \beta, \Omega} \leq \sqrt{2+1 / M}\|\phi\|_{\Omega}, \quad \forall \phi \in V_{M, N, \beta}(\Omega) .
\end{aligned}
$$

Let $\Omega_{M, N, \beta}=\left\{\left(\tilde{\xi}_{R, N, \Lambda, j}^{(\beta)}, \zeta_{L, M, I, k}\right), 0 \leq k \leq M, 0 \leq j \leq N\right\}$. The mixed Laguerre-Legendre interpolation $I_{M, N, \beta, \Omega} u \in V_{M, N, \beta}(\Omega)$ is determined by

$$
I_{M, N, \beta, \Omega} u(x, y)=u(x, y), \quad(x, y) \in \Omega_{M, N, \beta} .
$$

Then (cf. [24])

$$
\left\|I_{M, N, \beta, \Omega} u-u\right\|_{\Omega}^{2} \leq c\left(M^{-2 q}+(\beta N)^{1-r} \ln N\right) \mathbb{C}_{M, \beta, \Omega}^{q, r}(u),
$$


where

$$
\begin{aligned}
& \mathbb{C}_{M, \beta, \Omega}^{q, r}(u)=\int_{I}\left(\beta^{-2}\left\|\partial_{x}^{r}\left(e^{\frac{1}{2} \beta x} u\right)\right\|_{\omega_{r-1, \beta}, \Lambda}^{2}+\left(1+\beta^{-1}\right)\left\|\partial_{x}^{r}\left(e^{\frac{1}{2} \beta x} u\right)\right\|_{\omega_{r, \beta}, \Lambda}^{2}\right) d y \\
& \quad+M^{-2} \int_{I}\left(\beta^{-2}\left\|\partial_{x}^{r}\left(e^{\frac{1}{2} \beta x} \partial_{y} u\right)\right\|_{\omega_{r-1, \beta}, \Lambda}^{2}+\left(1+\beta^{-1}\right)\left\|\partial_{x}^{r}\left(e^{\frac{1}{2} \beta x} \partial_{y} u\right)\right\|_{\omega_{r, \beta}, \Lambda}^{2}\right) d y \\
& \quad+\int_{\Lambda}\left\|\left(1-y^{2}\right)^{\frac{q-1}{2}} \partial_{y}^{q} u\right\|_{I}^{2} d x
\end{aligned}
$$

with integers $r, q \geq 1$. Moreover, we have that for any $\phi \in V_{M, N, \beta}(\Omega)$ and integers $q, r \geq 1$ (cf. [24]),

$$
\left|(u, \phi)_{\Omega}-(u, \phi)_{M, N, \beta, \Omega}\right| \leq c\left(M^{-q}+(\beta N)^{\frac{1-r}{2}}(\ln N)^{\frac{1}{2}}\right)\left(\mathbb{C}_{M, \beta, \Omega}^{q, r}(u)\right)^{\frac{1}{2}}\|\phi\|_{\Omega} .
$$

\section{Mixed Laguerre-Legendre Pseudospectral Schemes}

In this section, we consider the mixed Laguerre-Legendre pseudospectral method for non-isotropic heat transfer in an infinite strip.

\subsection{Semi-discrete scheme}

Let $\Gamma=\{(x, y)|0<x<\infty| y \mid,=1\} \cup\{(x, y)|x=0| y \mid, \leq 1\}$ be the boundary of the domain $\Omega$ and $\bar{\Omega}=\Omega \cup \Gamma$. Denote by $W(x, y, t)$ the temperature. The positive constants $\nu$ and $\mu$ are the conductivities in the directions $x$ and $y$, respectively. The parameters $a$ and $b$ are some convective constants. Moreover, $F(x, y, t)$ and $W_{0}(x, y)$ describe the heat source and the initial state, respectively. For simplicity, let $\partial_{z} W=\frac{\partial W}{\partial z}$, etc. Then the nonisotropic heat transfer in an infinite strip is governed by

$$
\begin{cases}\partial_{t} W(x, y, t)+a \partial_{x} W(x, y, t)+b \partial_{y} W(x, y, t) & \\ -\nu \partial_{x}^{2} W(x, y, t)-\mu \partial_{y}^{2} W(x, y, t)=F(x, y, t), & (x, y) \in \Omega, 0<t \leq T \\ W(x, y, t)=W_{1}(x, y, t), & (x, y) \text { on } \Gamma, 0<t \leq T \\ W(x, y, t) \rightarrow 0, & x \rightarrow \infty, 0<t \leq T \\ W(x, y, 0)=W_{0}(x, y), & (x, y) \in \bar{\Omega}\end{cases}
$$

Let

$$
W_{1}(x, 1, t)=g_{1}(x, t), \quad W_{1}(0, y, t)=g_{2}(y, t), \quad W_{1}(x,-1, t)=g_{3}(x, t) .
$$

We assume that

$$
g_{1}(0, t)=g_{2}(1, t), \quad g_{2}(-1, t)=g_{3}(0, t)
$$

In fact, the above conditions implies that $W(x, y, t)$ is continuous at the two corners of boundary $\Gamma$. Next, set

$$
\begin{aligned}
W_{B}(x, y, t) & =\frac{1}{2} g_{1}(x, t)(1+y)+\frac{1}{2} g_{3}(x, t)(1-y)+e^{-\frac{1}{2} \beta x} g_{2}(y, t) \\
& -\frac{1}{2} e^{-\frac{1}{2} \beta x}(1-y) g_{3}(0, t)-\frac{1}{2} e^{-\frac{1}{2} \beta x}(1+y) g_{1}(0, t) .
\end{aligned}
$$


It can be checked that $W_{B}(x, y, t)=W(x, y, t)$ on $\Gamma$, and that $W_{B}(x, y, t) \rightarrow 0$ when $x \rightarrow \infty$. Now, we make a change of the variables $W(x, y, t)=U(x, y, t)+$ $W_{B}(x, y, t)$ and

$$
\begin{aligned}
f(x, y, t) & =F(x, y, t)-\partial_{t} W_{B}(x, y, t)-a \partial_{x} W_{B}(x, y, t) \\
& -b \partial_{y} W_{B}(x, y, t)+\nu \partial_{x}^{2} W_{B}(x, y, t)+\mu \partial_{y}^{2} W_{B}(x, y, t) .
\end{aligned}
$$

Then, (3.1) can be rewritten as

$$
\begin{cases}\partial_{t} U(x, y, t)+a \partial_{x} U(x, y, t)+b \partial_{y} U(x, y, t) & \\ -\nu \partial_{x}^{2} U(x, y, t)-\mu \partial_{y}^{2} U(x, y, t)=f(x, y, t), & (x, y) \in \Omega, 0<t \leq T \\ U(x, y, t)=0, & (x, y) \text { on } \Gamma, 0<t \leq T, \\ U(x, y, t) \rightarrow 0, & x \rightarrow \infty, 0<t \leq T, \\ U(x, y, 0)=U_{0}(x, y), & (x, y) \in \bar{\Omega},\end{cases}
$$

where $U_{0}(x, y)=W_{0}(x, y)-W_{B}(x, y, 0)$. We can follow the same idea as in [12] to prove the existence, uniqueness and regularity of (3.2).

Let $V_{M, N, \beta}^{0}(\Omega)=H_{0}^{1}(\Omega) \cap V_{M, N, \beta}(\Omega)$. A weak formulation of (3.2) is to seek solution $U \in L^{\infty}\left(0, T ; L^{2}(\Omega)\right) \cap L^{2}\left(0, T ; H_{0}^{1}(\Omega)\right)$, such that

$$
\left\{\begin{array}{l}
\left(\partial_{t} U(t), v\right)_{\Omega}+a\left(\partial_{x} U(t), v\right)_{\Omega}+b\left(\partial_{y} U(t), v\right)_{\Omega}+\nu\left(\partial_{x} U(t), \partial_{x} v\right)_{\Omega} \\
+\mu\left(\partial_{y} U(t), \partial_{y} v\right)_{\Omega}=(f(t), v)_{\Omega}, \quad \forall v \in H_{0}^{1}(\Omega), \quad 0<t \leq T \\
U(0)=U_{0}
\end{array}\right.
$$

The mixed pseudo-spectral scheme for (3.3) is to find $u_{M, N} \in V_{M, N, \beta}^{0}(\Omega)$, such that

$$
\left\{\begin{array}{c}
\left(\partial_{t} u_{M, N}(t), \phi\right)_{M, N, \beta, \Omega}+a\left(\partial_{x} u_{M, N}(t), \phi\right)_{M, N, \beta, \Omega}+b\left(\partial_{y} u_{M, N}(t), \phi\right)_{M, N, \beta, \Omega} \\
\quad+\nu\left(\partial_{x} u_{M, N}(t), \partial_{x} \phi\right)_{M, N, \beta, \Omega}+\mu\left(\partial_{y} u_{M, N}(t), \partial_{y} \phi\right)_{M, N, \beta, \Omega} \\
=(f(t), \phi)_{M, N, \beta, \Omega}, \quad \forall \phi \in V_{M, N, \beta}^{0}(\Omega), \quad 0<t \leq T, \\
u_{M, N}(0)=I_{M, N, \beta, \Omega} U_{0} .
\end{array}\right.
$$

Thanks to (2.6), (3.4) is equivalent to

$$
\left\{\begin{array}{c}
\left(\partial_{t} u_{M, N}(t), \phi\right)_{M, N, \beta, \Omega}+a\left(\partial_{x} u_{M, N}(t), \phi\right)_{M, N, \beta, \Omega}+b\left(\partial_{y} u_{M, N}(t), \phi\right)_{\Omega} \\
+\nu\left(\partial_{x} u_{M, N}(t), \partial_{x} \phi\right)_{M, N, \beta, \Omega}+\mu\left(\partial_{y} u_{M, N}(t), \partial_{y} \phi\right)_{\Omega}=(f(t), \phi)_{M, N, \beta, \Omega}, \\
\quad \forall \phi \in V_{M, N, \beta}^{0}(\Omega), \quad 0<t \leq T, \\
u_{M, N}(0)=I_{M, N, \beta, \Omega} U_{0} \quad
\end{array}\right.
$$

The numerical solution of problem (3.1) is given by

$$
w_{M, N}(t)=u_{M, N}(t)+W_{B}(x, y, t) .
$$

In order to analyze the numerical errors, we need to study the mixed Laguerre-Legendre approximation. We define the weighted space as follow

$$
H_{0, \omega_{\beta}}^{1}(\Omega)=\left\{v \mid v \in H_{\omega_{\beta}}^{1}(\Omega), v(0, y)=v(x, \pm 1)=0\right\} .
$$


Lemma 1. For any $v \in H_{0, \omega_{\beta}}^{1}(\Omega)$,

$$
\|v\|_{\omega_{\beta}, \Omega} \leq \frac{2}{\sqrt{\beta^{2}+2}}\|\nabla v\|_{\omega_{\beta}, \Omega} .
$$

Proof. For any $v \in H_{0, \omega_{\beta}}^{1}(\Omega)$, following the same line as in the derivation of Lemma 2.4 of [27], we can prove the following results,

$$
\beta\|v\|_{\omega_{\beta}, \Omega} \leq 2\left\|\partial_{x} v\right\|_{\omega_{\beta}, \Omega}, \quad\|v\|_{\omega_{\beta}, \Omega} \leq \sqrt{2}\left\|\partial_{y} v\right\|_{\omega_{\beta}, \Omega}
$$

the combination of the above two formulas leads to (3.7).

Lemma 2. For any $u \in H_{0}^{1}(\Omega)$,

$$
\|u\|_{\Omega} \leq \sqrt{2}\|\nabla u\|_{\Omega}
$$

Proof. For any $u \in H_{0}^{1}(\Omega)$,

$$
\int_{I} u^{2}(x, y) d y \rightarrow 0, \text { as } x \rightarrow \infty
$$

By (3.7) and integration by parts,

$$
\begin{aligned}
\|u\|_{\Omega}^{2} & =\left\|e^{\frac{1}{2} \beta x} u\right\|_{\omega_{\beta}, \Omega}^{2} \leq \frac{4}{\beta^{2}+2}\left\|\nabla\left(e^{\frac{1}{2} \beta x} u\right)\right\|_{\omega_{\beta}, \Omega}^{2} \\
& =\frac{4}{\beta^{2}+2} \iint_{\Omega} e^{-\beta x}\left(\left(\partial_{x}\left(e^{\frac{1}{2} \beta x} u\right)\right)^{2}+\left(\partial_{y}\left(e^{\frac{1}{2} \beta x} u\right)\right)^{2}\right) d x d y \\
& =\frac{4}{\beta^{2}+2} \iint_{\Omega} e^{-\beta x}\left(\left(\frac{\beta}{2} e^{\frac{1}{2} \beta x} u+e^{\frac{1}{2} \beta x} \partial_{x} u\right)^{2}+e^{\beta x}\left(\partial_{y} u\right)^{2}\right) d x d y \\
& =\frac{4}{\beta^{2}+2} \iint_{\Omega}\left(\left(\frac{\beta^{2}}{4} u^{2}+\beta u \partial_{x} u+\left(\partial_{x} u\right)^{2}+\left(\partial_{y} u\right)^{2}\right) d x d y\right. \\
& =\frac{4}{\beta^{2}+2} \iint_{\Omega}\left(\left(\frac{\beta^{2}}{4} u^{2}+\left(\partial_{x} u\right)^{2}+\left(\partial_{y} u\right)^{2}\right) d x d y,\right.
\end{aligned}
$$

which implies the desired result.

The orthogonal projection $P_{M, N, \Omega}^{1,0}: H_{0}^{1}(\Omega) \rightarrow V_{M, N, \beta}^{0}(\Omega)$ is defined by

$$
\left(\nabla\left(P_{M, N, \Omega}^{1,0} u-u\right), \nabla \phi\right)=0, \quad \forall \phi \in V_{M, N, \beta}^{0}(\Omega) .
$$

We shall use the follow notations with integers $q, r \geq 1$,

$$
\begin{aligned}
& \mathcal{B}_{\beta, \Omega}^{q, r}(u)=\left(1+\beta^{-2}\right) \\
& \quad \times \int_{I}\left(\left\|\partial_{x}^{r}\left(e^{\frac{1}{2} \beta x} u\right)\right\|_{\omega_{r-1, \beta}, \Lambda}^{2}+\left(1-y^{2}\right)^{q-1}\left\|\partial_{x}\left(e^{\frac{1}{2} \beta x} \partial_{y}^{q} u\right)\right\|_{\omega_{\beta}, \Lambda}^{2}\right) d y \\
& +\beta^{-2} \int_{I}\left\|\partial_{x}^{r}\left(e^{\frac{1}{2} \beta x} \partial_{y} u\right)\right\|_{\omega_{r-1, \beta}, \Lambda}^{2} d y+\sum_{k=0}^{1} \int_{\Lambda}\left\|\left(1-y^{2}\right)^{\frac{q-1}{2}} \partial_{x}^{k} \partial_{y}^{q} u\right\|_{I}^{2} d x
\end{aligned}
$$


Theorem 1. For any $u \in H_{0}^{1}(\Omega)$ and integers $1 \leq r \leq N+1$ and $1 \leq q \leq$ $M+1$,

$$
\left\|\nabla^{s}\left(P_{M, N, \beta, \Omega}^{1,0} u-u\right)\right\|_{\Omega}^{2} \leq c\left(M^{2-2 q}+(\beta N)^{1-r}\right) \mathcal{B}_{\beta, \Omega}^{q, r}(u), \quad s=0,1,
$$

provided that $\mathcal{B}_{\beta, \Omega}^{q, r}(u)$ is finite.

Proof. Clear, ${ }_{0} P_{N, \beta, \Lambda}^{1}\left(P_{M, I}^{1,0} u\right) \in V_{M, N, \beta}^{0}(\Omega)$. By projection theorem,

$$
\begin{aligned}
& \left\|\nabla\left(P_{M, N, \beta, \Omega}^{1,0} u-u\right)\right\|_{\Omega}^{2}=\inf _{\phi \in V_{M, N, \beta}^{0}(\Omega)}\|\nabla(\phi-u)\|_{\Omega}^{2} \\
& \quad \leq\left\|\nabla\left({ }_{0} P_{N, \beta, \Lambda}^{1}\left(P_{M, I}^{1,0} u\right)-u\right)\right\|_{\Omega}^{2} \leq F_{1}(u)+F_{2}(u)+F_{3}(u),
\end{aligned}
$$

where

$$
\begin{aligned}
& F_{1}(u)=2\left\|\partial_{x}\left({ }_{0} P_{N, \beta, \Lambda}^{1} u-u\right)\right\|_{\Omega}^{2}+2\left\|\partial_{y}\left({ }_{0} P_{N, \beta, \Lambda}^{1} u-u\right)\right\|_{\Omega}^{2}, \\
& F_{2}(u)=2\left\|\partial_{x}\left(P_{M, I}^{1,0}\left({ }_{0} P_{N, \beta, \Lambda}^{1} u\right)-{ }_{0} P_{N, \beta, \Lambda}^{1} u\right)\right\|_{\Omega}^{2}, \\
& F_{3}(u)=2\left\|\partial_{y}\left(P_{M, I}^{1,0}\left({ }_{0} P_{N, \beta, \Lambda}^{1} u\right)-{ }_{0} P_{N, \beta, \Lambda}^{1} u\right)\right\|_{\Omega}^{2} .
\end{aligned}
$$

Thanks to $(2.3)$ with $s=0,1$,

$$
\begin{aligned}
F_{1}(u) \leq & c(\beta N)^{1-r} \int_{I}\left\|\partial_{x}^{r}\left(e^{\frac{1}{2} \beta x} u\right)\right\|_{\omega_{r-1, \beta}, \Lambda}^{2} d y \\
& +c \beta^{-2}(\beta N)^{1-r} \int_{I}\left\|\partial_{x}^{r}\left(e^{\frac{1}{2} \beta x} \partial_{y} u\right)\right\|_{\omega_{r-1, \beta}, \Lambda}^{2} d y
\end{aligned}
$$

Next, by using (2.5) with $s=0$ and (2.3) with $s=1, r=1$, we obtain that

$$
\begin{aligned}
F_{2}(u) & \leq c M^{-2 q} \int_{\Lambda}\left\|\left(1-y^{2}\right)^{\frac{q-1}{2}} \partial_{x} \partial_{y}^{q}\left({ }_{0} P_{N, \beta, \Lambda}^{1} u\right)\right\|_{I}^{2} d x \\
& \leq c M^{-2 q} \int_{\Lambda}\left\|\left(1-y^{2}\right)^{\frac{q-1}{2}} \partial_{x} \partial_{y}^{q} u\right\|_{I}^{2} d x \\
& +c M^{-2 q} \int_{I}\left(1-y^{2}\right)^{q-1}\left\|\partial_{x}\left(e^{\frac{1}{2} \beta x} \partial_{y}^{q} u\right)\right\|_{\omega_{\beta}, \Lambda}^{2} d y .
\end{aligned}
$$

Using (2.5) with $s=1$ and (2.3) with $s=0, r=1$ again, we deduce that

$$
\begin{aligned}
F_{3}(u) & \leq c M^{2-2 q} \int_{\Lambda}\left\|\left(1-y^{2}\right)^{\frac{q-1}{2}} \partial_{y}^{q}\left({ }_{0} P_{N, \beta, \Lambda}^{1} u\right)\right\|_{I}^{2} d x \\
& \leq c M^{2-2 q} \int_{\Lambda}\left\|\left(1-y^{2}\right)^{\frac{q-1}{2}} \partial_{y}^{q} u\right\|_{I}^{2} d x \\
& +c \beta^{-2} M^{2-2 q} \int_{I}\left(1-y^{2}\right)^{q-1}\left\|\partial_{x}\left(e^{\frac{1}{2} \beta x} \partial_{y}^{q} u\right)\right\|_{\omega_{\beta}, \Lambda}^{2} d y .
\end{aligned}
$$

Finally, the desired results comes from a combination of previous statements and (3.8).

Remark 1. For the domain decomposition spectral method of exterior problems, we refer to the Theorem 2.3 of work [13], which is the quasi-orthogonal approximation. 
In order to analyze the numerical errors of non-isotropic heat transfer in an infinite strip, we need the next following orthogonal projection. To this end, we introduce the bilinear form $a_{\alpha, \gamma}(u, v)$ as

$$
a_{\alpha, \gamma}(u, v)=\alpha\left(\partial_{x} u, \partial_{x} v\right)_{\Omega}+\gamma\left(\partial_{y} u, \partial_{y} v\right)_{\Omega}, \quad \alpha, \gamma>0, \quad \forall u, v \in H_{0}^{1}(\Omega)
$$

The projection $P_{M, N, \beta, \alpha, \gamma, \Omega}^{1,0}: H_{0}^{1}(\Omega) \rightarrow V_{M, N, \beta}^{0}(\Omega)$ is defined by

$$
a_{\alpha, \gamma}\left(P_{M, N, \beta, \alpha, \gamma, \Omega}^{1,0} u-u, \phi\right)_{\Omega}=0, \quad \forall \phi \in V_{M, N, \beta}^{0}(\Omega) .
$$

By (3.8) and Theorem 1, we can prove the following results.

Theorem 2. For any $u \in H_{0}^{1}(\Omega)$, and integers $1 \leq r \leq N+1$ and $1 \leq q \leq$ $M+1$,

$$
\begin{aligned}
& \alpha\left\|\partial_{x}\left(P_{M, N, \beta, \alpha, \gamma, \Omega}^{1,0} u-u\right)\right\|_{\Omega}^{2}+\gamma\left\|\partial_{y}\left(P_{M, N, \beta, \alpha, \gamma, \Omega}^{1,0} u-u\right)\right\|_{\Omega}^{2} \\
& \leq c(\alpha+\gamma)\left(M^{2-2 q}+(\beta N)^{1-r}\right) \mathcal{B}_{\beta, \Omega}^{q, r}(u), \\
& \left\|P_{M, N, \beta, \alpha, \gamma, \Omega}^{1,0} u-u\right\|_{\Omega}^{2} \leq c(\alpha+\gamma)\left(M^{2-2 q}+(\beta N)^{1-r}\right) \mathcal{B}_{\beta, \Omega}^{q, r}(u) .
\end{aligned}
$$

We now deal with the convergence of scheme (3.5). Let $U_{M, N}=P_{M, N, \beta, \nu, \mu, \Omega}^{1,0} U$. By using (2.6) and (3.9), we derive from (3.3) that

$$
\begin{cases}\left(\partial_{t} U_{M, N}(t), \phi\right)_{M, N, \beta, \Omega}+a\left(\partial_{x} U_{M, N}(t), \phi\right)_{M, N, \beta, \Omega} \\ \quad+b\left(\partial_{y} U_{M, N}(t), \phi\right)_{\Omega}+\nu\left(\partial_{x} U_{M, N}(t), \partial_{x} \phi\right)_{M, N, \beta, \Omega}+\mu\left(\partial_{y} U_{M, N}(t), \partial_{y} \phi\right)_{\Omega} \\ \quad=\sum_{j=1}^{6} G_{j}(t, \phi)+(f(t), \phi)_{M, N, \beta, \Omega}, \quad \forall \phi \in V_{M, N, \beta}^{0}(\Omega), \quad 0<t \leq T, \\ U_{M, N}(0)=P_{M, N, \beta, \nu, \mu, \Omega}^{1,0} U_{0},\end{cases}
$$

where

$$
\begin{aligned}
& G_{1}(t, \phi)=\left(\partial_{t} U_{M, N}(t), \phi\right)_{M, N, \beta, \Omega}-\left(\partial_{t} U(t), \phi\right)_{\Omega}, \\
& G_{2}(t, \phi)=a\left(\partial_{x} U_{M, N}(t), \phi\right)_{M, N, \beta, \Omega}-a\left(\partial_{x} U(t), \phi\right)_{\Omega}, \\
& G_{3}(t, \phi)=b\left(\partial_{y}\left(U_{M, N}(t)-U(t)\right), \phi\right)_{\Omega}, \\
& G_{4}(t, \phi)=\nu\left(\partial_{x} U_{M, N}(t), \partial_{x} \phi\right)_{M, N, \beta, \Omega}-\nu\left(\partial_{x} U(t), \partial_{x} \phi\right)_{\Omega}, \\
& G_{5}(t, \phi)=\mu\left(\partial_{y}\left(U_{M, N}(t)-U(t)\right), \partial_{y} \phi\right)_{\Omega}, \\
& G_{6}(t, \phi)=(f, \phi)_{\Omega}-(f, \phi)_{M, N, \beta, \Omega} .
\end{aligned}
$$

Taking $\widetilde{U}_{M, N}=u_{M, N}-U_{M, N}$ and subtracting (3.11) from (3.5), we obtain that

$$
\left\{\begin{array}{c}
\left(\partial_{t} \widetilde{U}_{M, N}(t), \phi\right)_{M, N, \beta, \Omega}+a\left(\partial_{x} \widetilde{U}_{M, N}(t), \phi\right)_{M, N, \beta, \Omega}+b\left(\partial_{y} \widetilde{U}_{M, N}(t), \phi\right)_{\Omega} \\
+\nu\left(\partial_{x} \widetilde{U}_{M, N}(t), \partial_{x} \phi\right)_{M, N, \beta, \Omega}+\mu\left(\partial_{y} \widetilde{U}_{M, N}(t), \partial_{y} \phi\right)_{\Omega}=-\sum_{j=1}^{6} G_{j}(t, \phi), \\
\forall \phi \in V_{M, N, \beta}^{0}(\Omega), \quad 0<t \leq T \\
\widetilde{U}_{M, N}(0)=I_{M, N, \beta, \Omega} U_{0}-P_{M, N, \beta, \nu, \mu, \Omega}^{1,0} U_{0} .
\end{array}\right.
$$


Take $\phi=2 \tilde{U}_{M, N}$ in (3.12). We deduce that for $0<t \leq T$

$$
\begin{gathered}
\partial_{t}\left\|\widetilde{U}_{M, N}(t)\right\|_{M, N, \beta, \Omega}^{2}+2 \nu\left\|\partial_{x} \widetilde{U}_{M, N}(t)\right\|_{M, N, \beta, \Omega}^{2} \\
+2 \mu\left\|\partial_{y} \widetilde{U}_{M, N}(t)\right\|_{\Omega}^{2}=-2 \sum_{j=1}^{7} G_{j}(t, \phi),
\end{gathered}
$$

where $G_{7}(t, \phi)=a\left(\partial_{x} \widetilde{U}_{M, N}, \widetilde{U}_{M, N}\right)_{M, N, \beta, \Omega}$. Therefore, it suffices to estimate the terms $2\left|G_{j}\left(t, \widetilde{U}_{M, N}\right)\right|$. Firstly, we use the Cauchy inequality, (2.6), (2.7) and (3.10) to verify that for integers $r, q \geq 1$,

$$
\begin{aligned}
& 2\left|G_{1}\left(t, \tilde{U}_{M, N}(t)\right)\right| \\
& \quad=2 \mid\left(\partial_{t} U_{M, N}(t)-P_{M-1, N, \beta, \nu, \mu, \Omega}^{1,0} \partial_{t} U(t), \tilde{U}_{M, N}(t)\right)_{M, N, \beta, \Omega} \\
& \quad+\left(P_{M-1, N, \beta, \nu, \mu, \Omega}^{1,0} \partial_{t} U(t)-\partial_{t} U(t), \phi\right)_{\Omega} \mid \\
& \quad \leq 2\left(\sqrt{3}|| \partial_{t}\left(U(t)-U_{M-1, N}(t)\right) \|\left.\right|_{\Omega}\right. \\
& \left.\quad+\left\|\partial_{t} U(t)-P_{M-1, N, \beta, \nu, \mu, \Omega}^{1,0} \partial_{t} U(t)\right\|_{\Omega}\right)\left\|\tilde{U}_{M, N}(t)\right\|_{\Omega} \\
& \quad \leq c(\nu+\mu)\left(M^{2-2 q}+(\beta N)^{1-r}\right) \mathcal{B}_{\beta, \Omega}^{q, r}\left(\partial_{t} U(t)\right)+\left\|\tilde{U}_{M, N}(t)\right\|_{\Omega}^{2}
\end{aligned}
$$

Similarly,

$$
\begin{aligned}
& 2\left|G_{2}\left(t, \tilde{U}_{M, N}(t)\right)+G_{3}\left(t, \tilde{U}_{M, N}(t)\right)+G_{4}\left(t, \tilde{U}_{M, N}(t)\right)+G_{5}\left(t, \tilde{U}_{M, N}(t)\right)\right| \\
& \leq c\left(\frac{1}{\nu}+\frac{1}{\mu}+1\right)(\nu+\mu)\left(M^{2-2 q}+(\beta N)^{1-r}\right)\left(\mathcal{B}_{\beta, \Omega}^{q, r}(U(t))+\mathcal{B}_{\beta, \Omega}^{q, r}\left(\partial_{x} U(t)\right)\right) \\
& \quad+\frac{1}{2} \nu\left\|\partial_{x} \tilde{U}_{M, N}(t)\right\|_{\Omega}^{2}+\mu\left\|\partial_{y} \tilde{U}_{M, N}(t)\right\|_{\Omega}^{2}+\left(a^{2}+b^{2}\right)\left\|\tilde{U}_{M, N}(t)\right\|_{\Omega}^{2} .
\end{aligned}
$$

Obviously,

$$
2\left|G_{6}\left(t, \widetilde{U}_{M, N}\right)\right| \leq c\left(M^{2-2 q}+(\beta N)^{1-r} \ln N\right) \mathbb{C}_{M, \beta, \Omega}^{q-1, r}(f(t))+\left\|\tilde{U}_{M, N}(t)\right\|_{\Omega}^{2} .
$$

Next, by (2.7) and Cauchy inequality,

$$
\begin{gathered}
2\left|G_{7}\left(t, \widetilde{U}_{M, N}\right)\right| \leq 6|a|\left\|\partial_{x} \tilde{U}_{M, N}(t)\right\|\left\|_{\Omega}\right\| \tilde{U}_{M, N}(t) \|_{\Omega} \\
\leq \frac{1}{2} \nu\left\|\partial_{x} \tilde{U}_{M, N}(t)\right\|_{\Omega}^{2}+\frac{18 a^{2}}{\nu}\left\|\tilde{U}_{M, N}(t)\right\|_{\Omega}^{2} .
\end{gathered}
$$

Furthermore, we use (2.9) and (3.10) to derive that

$$
\begin{aligned}
\left\|\widetilde{U}_{M, N}(0)\right\|_{\Omega}^{2} & \leq 2\left\|I_{M, N, \beta, \Omega} U_{0}-U_{0}\right\|_{\Omega}^{2}+2\left\|U_{0}-P_{M, N, \beta, \nu, \mu, \Omega}^{1,0} U_{0}\right\|_{\Omega}^{2} \\
& \leq c(\nu+\mu)\left(M^{2-2 q}+(\beta N)^{1-r} \ln N\right)\left(\mathcal{B}_{\beta, \Omega}^{q, r}\left(U_{0}\right)+\mathbb{C}_{\beta, \Omega}^{q-1, r}\left(U_{0}\right)\right) .
\end{aligned}
$$

For simplicity of statements, let

$$
\begin{aligned}
& E_{M, N, \nu, \mu, \beta}(u(t))=\|u(t)\|_{M, N, \beta, \Omega}^{2}+\int_{0}^{t}\left(\nu\left\|\partial_{x} u(\xi)\right\|_{M, N, \beta, \Omega}^{2}+\mu\left\|\partial_{y} u(\xi)\right\|_{\Omega}^{2}\right) d \xi . \\
& E_{\nu, \mu, \beta}(u(t))=\|u(t)\|_{\Omega}^{2}+\int_{0}^{t}\left(\nu\left\|\partial_{x} u(\xi)\right\|_{\Omega}^{2}+\mu\left\|\partial_{y} u(\xi)\right\|_{\Omega}^{2}\right) d \xi .
\end{aligned}
$$

By inserting (3.14)-(3.17) into (3.13), we find that

$$
\begin{aligned}
& \partial_{t} E_{M, N, \nu, \mu, \beta}\left(\widetilde{U}_{M, N}(t)\right) \\
& \quad \leq d^{*} E_{\nu, \mu}\left(\widetilde{U}_{M, N}(t)\right)+c^{*}\left(M^{2-2 q}+(\beta N)^{1-r} \ln N\right) \mathbb{D}_{\beta, \Omega}^{q, r}(t),
\end{aligned}
$$


where

$$
\begin{aligned}
& c^{*}=c\left(\frac{1}{\nu}+\frac{1}{\mu}+1\right)(\nu+\mu), \quad d^{*}=\left(1+\frac{18}{\nu}\right) a^{2}+b^{2}+2, \\
& \mathbb{D}_{\beta, \Omega}^{q, r}(t)=\mathcal{B}_{\beta, \Omega}^{q, r}\left(\partial_{t} U(t)\right)+\mathcal{B}_{\beta, \Omega}^{q, r}(U(t))+\mathcal{B}_{\beta, \Omega}^{q, r}\left(\partial_{x} U(t)\right)+\mathbb{C}_{M, \beta, \Omega}^{q-1, r}(f(t)) .
\end{aligned}
$$

Due to $(2.7)$, we have that

$$
E_{\nu, \mu, \beta}\left(\widetilde{U}_{M, N}(t)\right) \leq E_{M, N, \nu, \mu, \beta}\left(\widetilde{U}_{M, N}(t)\right) \leq c E_{\nu, \mu, \beta}\left(\widetilde{U}_{M, N}(t)\right) .
$$

Then (3.18) reads

$$
\begin{aligned}
& \partial_{t}\left(E_{M, N, \nu, \mu, \beta}\left(\widetilde{U}_{M, N}(t)\right) e^{-d^{*} t}\right) \\
& \quad \leq c c^{*} e^{-d^{*} t}\left(M^{2-2 q}+(\beta N)^{1-r} \ln N\right) \mathbb{D}_{\beta, \Omega}^{q, r}(t) .
\end{aligned}
$$

Integrating (3.20) with respect to $t$ and using (3.19), we obtain that

$$
\begin{aligned}
E_{\nu, \mu, \beta,}\left(\widetilde{U}_{M, N}(t)\right) & \leq E_{M, N, \nu, \mu, \beta}\left(\widetilde{U}_{M, N}(t)\right) \\
& \leq c\left(M^{2-2 q}+(\beta N)^{1-r}\right) R_{\Omega}\left(U, U_{0}, q, r, \nu, \mu, \beta, t\right),
\end{aligned}
$$

where

$$
\begin{aligned}
& R_{\Omega}\left(U, U_{0}, q, r, \nu, \mu, \beta, t\right) \\
& \quad=e^{d^{*} t}\left(c^{*} \int_{0}^{t} e^{-d^{*} \xi} \mathbb{D}_{\beta, \Omega}^{q, r}(\xi) d \xi+(\nu+\mu)\left(\mathcal{B}_{\beta, \Omega}^{q, r}\left(U_{0}\right)+\mathbb{C}_{M, \beta, \Omega}^{q-1, r}\left(U_{0}\right)\right)\right) .
\end{aligned}
$$

A combination of (3.10) and (3.21) leads to that

$$
\begin{aligned}
& E_{\nu, \mu}\left(U(t)-u_{M, N}(t)\right) \leq c\left(M^{2-2 q}+(\beta N)^{1-r} \ln N\right) \\
& \quad \cdot\left(R_{\Omega}\left(U, U_{0}, q, r, \nu, \mu, \beta, t\right)+(\nu+\mu)\left(\int_{0}^{t} \mathcal{B}_{\beta, \Omega}^{q, r}(U(\xi)) d \xi+\mathcal{B}_{\beta, \Omega}^{q, r}(U(t))\right)\right) .
\end{aligned}
$$

This, together with (3.6), implies that for $0 \leq t \leq T$,

$$
\begin{aligned}
& E_{\nu, \mu}\left(W(t)-w_{M, N}(t)\right) \\
& \quad \leq c\left(M^{2-2 q}+(\beta N)^{1-r} \ln N\right)\left(\bar{R}_{\Omega}\left(W, W_{0}, W_{B}, q, r, \nu, \mu, \beta, t\right)\right. \\
& \quad+(\nu+\mu)\left(\int_{0}^{t}\left(\mathcal{B}_{\beta, \Omega}^{q, r}(W(\xi))+\mathcal{B}_{\beta, \Omega}^{q, r}\left(W_{B}(\xi)\right)\right) d \xi\right. \\
& \left.\left.\quad+\mathcal{B}_{\beta, \Omega}^{q, r}(W(t))+\mathcal{B}_{\beta, \Omega}^{q, r}\left(W_{B}(t)\right)\right)\right),
\end{aligned}
$$

where

$$
\begin{aligned}
& \bar{R}_{\Omega}\left(W, W_{0}, W_{B}, q, r, \nu, \mu, \beta, t\right) \\
& \quad=e^{d^{*} t}\left(c^{*} \int_{0}^{t} e^{-d^{*} \xi} \mathcal{D}_{\beta, \Omega}^{q, r}(\xi) d \xi+(\nu+\mu)\left(\mathcal{B}_{\beta, \Omega}^{q, r}\left(W_{0}\right)+\mathbb{C}_{M, \beta, \Omega}^{q-1, r}\left(W_{0}\right)\right.\right. \\
& \left.\left.\quad+\mathcal{B}_{\beta, \Omega}^{q, r}\left(W_{B}(\cdot, \cdot, 0)\right)+\mathbb{C}_{M, \beta, \Omega}^{q-1, r}\left(W_{B}(\cdot, \cdot, 0)\right)\right)\right)
\end{aligned}
$$

with

$$
\begin{aligned}
& \mathcal{D}_{\beta, \Omega}^{q, r}(t)=\mathcal{B}_{\beta, \Omega}^{q, r}\left(\partial_{t} W(t)\right)+\mathcal{B}_{\beta, \Omega}^{q, r}(W(t))+\mathcal{B}_{\beta, \Omega}^{q, r}\left(\partial_{x} W(t)\right)+\mathbb{C}_{M, \beta, \Omega}^{q-1, r}(F(t)) \\
& \quad+\mathcal{B}_{\beta, \Omega}^{q, r}\left(\partial_{t} W_{B}(t)\right)+\mathcal{B}_{\beta, \Omega}^{q, r}\left(W_{B}(t)\right)+\mathcal{B}_{\beta, \Omega}^{q, r}\left(\partial_{x} W_{B}(t)\right)+\mathbb{C}_{M, \beta, \Omega}^{q-1, r}\left(\partial_{t} W_{B}(t)\right) \\
& \quad+\mathbb{C}_{M,-1, r}^{q-1, \Omega}\left(a \partial_{x} W_{B}(t)\right)+\mathbb{C}_{M, \beta, \Omega}^{q-1, r}\left(b \partial_{y} W_{B}(t)\right)+\mathbb{C}_{M, \beta, \Omega}^{q-1, r}\left(\nu \partial_{x}^{2} W_{B}(t)\right) \\
& \quad+\mathbb{C}_{M, \beta, \Omega}^{q-1, r}\left(\mu \partial_{y}^{2} W_{B}(t)\right) .
\end{aligned}
$$

Remark 2. The result (3.10) improve the result (3.5) of [25]. In fact, the right term of (3.5) of [25] includes the factor $(\alpha+\gamma+1)$. 


\subsection{Full-discrete scheme}

We consider the full-discrete scheme of (3.2). Denote by $[T]$ the integer part of any fixed positive constant $T$. Let $\tau$ be the mesh size of the variable $t$ and

$$
R_{\tau}(T)=\{t=k \tau \mid k=1,2, \cdots,[T / \tau]\}, \quad \bar{R}_{\tau}(T)=R_{\tau}(T) \cup\{0\} .
$$

Let

$$
D_{\tau} u(t)=\frac{1}{\tau}(u(t+\tau)-u(t)), \quad \hat{u}(t)=\frac{1}{2}(u(t+\tau)+u(t)) .
$$

In the numerical analysis of the full-discrete scheme of (3.2), we need the following approximation results.

Lemma 3. We have (cf. Lemma 4.6 of [7])

$$
\begin{aligned}
& \|\hat{u}(t)-u(t+\tau / 2)\|_{R} \leq c \tau^{\frac{3}{2}}\|u\|_{H^{2}\left(t, t+\tau ; L^{2}(R)\right)}, \\
& \left\|D_{\tau} u(t)-\partial_{t} u(t+\tau / 2)\right\|_{R} \leq c \tau^{\frac{3}{2}}\|u\|_{H^{3}\left(t, t+\tau ; L^{2}(R)\right)},
\end{aligned}
$$

provided that the related norms exist.

The full-discrete scheme of (3.2) is to seek $u_{M, N}(t) \in V_{M, N, \beta}^{0}(\Omega)$, such that

$$
\left\{\begin{array}{l}
\left(D_{\tau} u_{M, N}(t), \phi\right)_{M, N, \beta, \Omega}+a\left(\partial_{x} \hat{u}_{M, N}(t), \phi\right)_{M, N, \beta, \Omega}+b\left(\partial_{y} \hat{u}_{M, N}(t), \phi\right)_{\Omega} \\
\quad+\nu\left(\partial_{x} \hat{u}_{M, N}(t), \partial_{x} \phi\right)_{M, N, \beta, \Omega}+\mu\left(\partial_{y} \hat{u}_{M, N}(t), \partial_{y} \phi\right)_{\Omega}=(f(t), \phi)_{M, N, \beta, \Omega}, \\
\quad \forall \phi \in V_{M, N, \beta}^{0}(\Omega), \quad t \in R_{\tau}(T-\tau), \\
u_{M, N}(x, y, 0)=I_{M, N, \beta, \Omega} U_{0} .
\end{array}\right.
$$

We now consider the convergence of scheme (3.22). Let $U_{M, N}=P_{M, N, \beta, \nu, \mu, \Omega}^{1,0} U$. We derive from (3.3) that

$$
\left\{\begin{array}{l}
\quad\left(D_{\tau} U_{M, N}(t), \phi\right)_{M, N, \beta, \Omega}+a\left(\partial_{x} \hat{U}_{M, N}(t), \phi\right)_{M, N, \beta, \Omega}+b\left(\partial_{y} \hat{U}_{M, N}(t), \phi\right)_{\Omega} \\
\quad+\nu\left(\partial_{x} \hat{U}_{M, N}(t), \partial_{x} \phi\right)_{M, N, \beta, \Omega}+\mu\left(\partial_{y} \hat{U}_{M, N}(t), \partial_{y} \phi\right)_{\Omega} \\
=\sum_{j=j}^{6} \bar{G}_{j}(t, \phi)+(\hat{f}(t), \phi)_{M, N, \beta, \Omega}, \quad \forall \phi \in V_{M, N, \beta}^{0}(\Omega), \quad t \in R_{\tau}(T-\tau), \\
U_{M, N}(x, y, 0)=P_{M, N, \beta, \nu, \mu, \Omega}^{1,0} U_{0}(x, y),
\end{array}\right.
$$

where

$$
\begin{aligned}
\bar{G}_{1}(t, \phi) & =\left(D_{\tau} U_{M, N}(t), \phi\right)_{M, N, \beta, \Omega}-\left(\partial_{t} U(t+\tau / 2), \phi\right)_{\Omega} \\
\bar{G}_{2}(t, \phi) & =a\left(\partial_{x} \hat{U}_{M, N}(t), \phi\right)_{M, N, \beta, \Omega}-a\left(\partial_{x} U(t+\tau / 2), \phi\right)_{\Omega} \\
\bar{G}_{3}(t, \phi) & =b\left(\partial_{y}\left(\hat{U}_{M, N}(t)-U(t+\tau / 2)\right), \phi\right)_{\Omega} \\
\bar{G}_{4}(t, \phi) & =\nu\left(\partial_{x} \hat{U}_{M, N}(t), \partial_{x} \phi\right)_{M, N, \beta, \Omega}-\nu\left(\partial_{x} U(t+\tau / 2), \partial_{x} \phi\right)_{\Omega}, \\
\bar{G}_{5}(t, \phi) & =\mu\left(\partial_{y}\left(\hat{U}_{M, N}(t)-U(t+\tau / 2)\right), \partial_{y} \phi\right)_{\Omega} \\
\bar{G}_{6}(t, \phi) & =(f(t+\tau / 2), \phi)_{\Omega}-(\hat{f}(t), \phi)_{M, N, \beta, \Omega} .
\end{aligned}
$$

Taking $\widetilde{U}_{M, N}=u_{M, N}-U_{M, N}$ and subtracting (3.23) from (3.22), we obtain 
that

$$
\left\{\begin{array}{l}
\left(D_{\tau} \widetilde{U}_{M, N}(t), \phi\right)_{M, N, \beta, \Omega}+a\left(\partial_{x} \hat{\widetilde{U}}_{M, N}(t), \phi\right)_{M, N, \beta, \Omega}+b\left(\partial_{y} \hat{\widetilde{U}}_{M, N}(t), \phi\right)_{\Omega} \\
\quad+\nu\left(\partial_{x} \hat{\widetilde{U}}_{M, N}(t), \partial_{x} \phi\right)_{M, N, \beta, \Omega}+\mu\left(\partial_{y} \tilde{\widetilde{U}}_{M, N}(t), \partial_{y} \phi\right)_{\Omega} \\
=-\sum_{j=1}^{6} \bar{G}_{j}(t, \phi), \quad \forall \phi \in V_{M, N, \beta}^{0}(\Omega), \quad t \in R_{\tau}(T-\tau) \\
\widetilde{U}_{M, N}(x, y, 0)=I_{M, N, \beta, \Omega} U_{0}(x, y)-P_{M, N, \beta, \nu, \mu, \Omega}^{1,0} U_{0}(x, y) .
\end{array}\right.
$$

Taking $\phi=2 \hat{\tilde{U}}_{M, N}(t)$ in (3.24), we obtain that

$$
\begin{gathered}
D_{\tau}\left\|\widetilde{U}_{M, N}(t)\right\|_{M, N, \beta, \Omega}^{2}+2 \nu\left\|\partial_{x} \hat{\widetilde{U}}_{M, N}(t)\right\|_{M, N, \beta, \Omega}^{2} \\
+2 \mu\left\|\partial_{y} \hat{\tilde{U}}_{M, N}(t)\right\|_{\Omega}^{2}=-2 \sum_{j=1}^{8} \bar{G}_{j}(t, \phi),
\end{gathered}
$$

where

$$
\begin{aligned}
\bar{G}_{7}(t, \phi) & =a\left(\partial_{x} \hat{\widetilde{U}}_{M, N}(t), \hat{\widetilde{U}}_{M, N}(t)\right)_{M, N, \beta, \Omega}, \\
\bar{G}_{8}(t, \phi) & =b\left(\partial_{y} \hat{\widetilde{U}}_{M, N}(t), \hat{\widetilde{U}}_{M, N}(t)\right)_{\Omega} .
\end{aligned}
$$

Clearly,

$$
\begin{aligned}
& \left|\bar{G}_{1}\left(s, \hat{\tilde{U}}_{M, N}(s)\right)\right| \leq\left|\left(D_{\tau} U(t), \hat{\tilde{U}}_{M, N}(s)\right)_{\Omega}-\left(\partial_{t} U\left(t+\frac{\tau}{2}\right), \hat{\tilde{U}}_{M, N}(s)\right)_{\Omega}\right| \\
& \quad+\left|\left(D_{\tau} U(t), \hat{\tilde{U}}_{M, N}(s)\right)_{M, N, \beta, \Omega}-\left(D_{\tau} U(t), \hat{\tilde{U}}_{M, N}(s)\right)_{\Omega}\right| \\
& \quad+\left|\left(D_{\tau} U_{M, N}(t), \hat{\tilde{U}}_{M, N}(s)\right)_{M, N, \beta, \Omega}-\left(D_{\tau} U(t), \hat{\tilde{U}}_{M, N}(s)\right)_{M, N, \beta, \Omega}\right| .
\end{aligned}
$$

We use the Cauchy-Schwarz, Young's inequality, Lemma 3, (3.10), (2.8) and (2.10) to verify that for integers $q \geq 1, r \geq 1$ and $s \in R_{\tau}(t-\tau)$,

$$
\begin{aligned}
& 2 \tau\left|\bar{G}_{1}\left(s, \hat{\tilde{U}}_{M, N}(s)\right)\right| \leq \tau^{2}\left\|\hat{\tilde{U}}_{M, N}(s)\right\|_{\Omega}^{2}+c \tau^{4}\|U\|_{H^{3}\left(s, s+\tau ; L^{2}(\Omega)\right)}^{2} \\
& \quad+c(\nu+\mu)\left(M^{2-2 q}+(\beta N)^{1-r}\right) \mathcal{B}_{\beta, \Omega}^{q, r}\left(D_{\tau} U(s)\right) \\
& \quad+c\left(M^{-2 q}+(\beta N)^{1-r} \ln N\right) \mathbb{C}_{M, \beta, \Omega}^{q, r}\left(D_{\tau} U(s)\right) .
\end{aligned}
$$

Similarly,

$$
\begin{aligned}
& 2 \tau\left|\sum_{j=2}^{5} \bar{G}_{j}\left(s, \hat{\tilde{U}}_{M, N}(s)\right)\right| \leq c(\nu+\mu)\left(M^{2-2 q}+(\beta N)^{1-r}\right) \mathcal{B}_{\beta, \Omega}^{q, r}(\hat{U}(s)) \\
& \quad+\tau^{2}\left\|\hat{\tilde{U}}_{M, N}(s)\right\|_{\Omega}^{2}+c\left(M^{-2 q}+(\beta N)^{1-r} \ln N\right) \mathbb{C}_{M, \beta, \Omega}^{q, r}(\hat{U}(s)), \\
& 2 \tau\left|\bar{G}_{6}\left(s, \hat{\tilde{U}}_{M, N}(s)\right)\right| \leq c \tau^{4}\|f\|_{H^{2}\left(s, s+\tau ; L^{2}(\Omega)\right)}^{2}+\tau\left\|\hat{\tilde{U}}_{M, N}(s)\right\|_{\Omega}^{2} \\
& \quad+c\left(M^{-2 q}+(\beta N)^{1-r} \ln N\right) \mathbb{C}_{M, \beta, \Omega}^{q, r}(\hat{f}(s))+\tau^{2}\left\|\hat{\tilde{U}}_{M, N}(s)\right\|_{\Omega}^{2}, \\
& 2 \tau\left|\sum_{j=7}^{8} \bar{G}_{j}\left(s, \hat{\tilde{U}}_{M, N}(s)\right)\right| \leq \nu \tau\left\|\partial_{x} \hat{\tilde{U}}_{M, N}(s)\right\|_{\Omega}^{2}+\mu \tau\left\|\partial_{y} \hat{\tilde{U}}_{M, N}(s)\right\|_{\Omega}^{2} \\
& \quad+\tau\left(a^{2} / \nu+b^{2} / \mu\right)\left\|\hat{\tilde{U}}_{M, N}(s)\right\|_{\Omega}^{2} .
\end{aligned}
$$


Furthermore, we use (2.9) and (3.10) to derive that

$$
\begin{gathered}
\left\|\widetilde{U}_{M, N}(0)\right\|_{\Omega}^{2} \leq c(\nu+\mu)\left(M^{2-2 q}+(\beta N)^{1-r}\right) \mathcal{B}_{\beta, \Omega}^{q, r}\left(U_{0}\right) \\
+c\left(M^{-2 q}+(\beta N)^{1-r} \ln N\right) \mathbb{C}_{M, \beta, \Omega}^{q, r}\left(U_{0}(s)\right) .
\end{gathered}
$$

We sum up the (3.25) for $s \in \bar{R}_{\tau}(t-\tau)$, and use the (3.26)-(3.30) to obtain that

$$
\begin{aligned}
& \left(1-\tau\left(3 T+1+a^{2} / \nu+b^{2} / \mu\right)\left\|\widetilde{U}_{M, N}(t)\right\|_{M, N, \beta, \Omega}^{2}\right. \\
& +\tau \sum_{s \in \overline{\tilde{R}}_{\tau}(t-\tau)}\left(\nu\left\|\partial_{x} \hat{\widetilde{U}}_{M, N}(s)\right\|_{M, N, \beta, \Omega}^{2}+\mu\left\|\partial_{y} \tilde{\widetilde{U}}_{M, N}(s)\right\|_{\Omega}^{2}\right) \\
& \leq \tau \sum_{s \in \bar{R}_{\tau}(t-\tau)}\left(3 T+1+a^{2} / \nu+b^{2} / \mu\right)\left\|\widetilde{U}_{M, N}(s)\right\|_{M, N, \beta, \Omega}^{2} \\
& \quad+c \tau^{4}\left(\|U\|_{H^{3}\left(0, T ; L^{2}(\Omega)\right)}^{2}+\|f\|_{H^{2}\left(0, T ; L^{2}(\Omega)\right)}^{2}\right) \\
& \quad+c(\nu+\mu)\left(M^{2-2 q}+(\beta N)^{1-r} \ln N\right) \\
& \quad \times \sum_{s \in \bar{R}_{\tau}(t-\tau)}\left(\mathcal{B}_{\beta, \Omega}^{q, r}(\hat{U}(s))+\mathcal{B}_{\beta, \Omega}^{q, r}\left(D_{\tau} \hat{U}(s)\right)+\mathcal{B}_{\beta, \Omega}^{q, r}\left(U_{0}\right)+\mathbb{C}_{M, \beta, \Omega}^{q-1, r}(\hat{U}(s))\right. \\
& \left.\quad+\mathbb{C}_{M, \beta, \Omega}^{q-1, r}\left(D_{\tau} \hat{U}(s)\right)+\mathbb{C}_{M, \beta, \Omega}^{q-1, r}\left(U_{0}\right)+\mathbb{C}_{M, \beta, \Omega}^{q-1, r}(\hat{f}(s))\right) .
\end{aligned}
$$

Let

$$
\begin{aligned}
& E_{M, N, \beta, \Omega}(v, t) \\
& =\|v(t)\|_{M, N, \beta, \Omega}^{2}+\tau \sum_{s \in \bar{R}_{\tau}(t-\tau)}\left(\nu\left\|\partial_{x} \hat{v}(s)\right\|_{M, N, \beta, \Omega}^{2}+\mu \| \partial_{y}\left(\hat{v}(s) \|_{\Omega}^{2}\right),\right. \\
& \rho(t)=(\nu+\mu) \sum_{s \in \bar{R}_{\tau}(t-\tau)}\left(\mathcal{B}_{\beta, \Omega}^{q, r}(\hat{U}(s))+\mathcal{B}_{\beta, \Omega}^{q, r}\left(D_{\tau} \hat{U}(s)\right)+\mathcal{B}_{\beta, \Omega}^{q, r}\left(U_{0}\right)\right. \\
& \left.+\mathbb{C}_{M, \beta, \Omega}^{q-1, r}(\hat{U}(s))+\mathbb{C}_{M, \beta, \Omega}^{q-1, r}\left(D_{\tau} \hat{U}(s)\right)+\mathbb{C}_{M, \beta, \Omega}^{q-1, r}\left(U_{0}\right)+\mathbb{C}_{M, \beta, \Omega}^{q-1, r}(\hat{f}(s))\right) \\
& +\|U\|_{H^{3}\left(0, T ; L^{2}(\Omega)\right)}^{2}+\|f\|_{H^{2}\left(0, T ; L^{2}(\Omega)\right)}^{2} .
\end{aligned}
$$

Finally, using Lemma 2 of [7] for (3.31), we can derive that, if

$$
\tau<\left(3 T+1+a^{2} / \nu+b^{2} / \mu\right)^{-1},
$$

then for all $t \in \bar{R}_{\tau}(T)$

$$
\begin{aligned}
& E_{M, N, \beta, \Omega}\left(U_{M, N}-u_{M, N}, t\right) \\
& \quad \leq c \rho(t) e^{\left(3 T+1+\frac{a^{2}}{\nu}+\frac{b^{2}}{\mu}\right) t}\left(\tau^{4}+M^{2-2 q}+(\beta N)^{1-r} \ln N\right) .
\end{aligned}
$$

\section{Numerical Results}

In this section, we describe the implementations for pseudospectral schemes (3.5), and present some numerical results confirming the theoretical analysis. We use the Crank-Nicolson discretization in time $t$, with the mesh size $\tau$.

For simplicity of statements, we use the notation

$$
\begin{aligned}
a_{M, N, \Omega}(z, \phi)= & a\left(\partial_{x} z, \phi\right)_{M, N, \beta, \Omega}+b\left(\partial_{y} z, \phi\right)_{\Omega} \\
& +\nu\left(\partial_{x} z, \partial_{x} \phi\right)_{M, N, \beta, \Omega}+\mu\left(\partial_{y} z, \partial_{y} \phi\right)_{\Omega} .
\end{aligned}
$$


Then the fully discrete scheme of (3.22) reads,

$$
\left\{\begin{array}{l}
\frac{1}{\tau}\left(u_{M, N}(t+\tau)-u_{M, N}(t), \phi\right)_{M, N, \beta, \Omega}+\frac{1}{2} a_{M, N, \Omega}\left(u_{M, N}(t+\tau)+u_{M, N}(t), \phi\right) \\
=\frac{1}{2}(f(t+\tau)+f(t), \phi)_{M, N, \beta, \Omega}, \quad t=0, \tau, 2 \tau, \cdots, T-\tau, \\
u_{M, N}(0)=P_{M, N, \beta, \Omega} U_{0} .
\end{array}\right.
$$

Let

$$
\begin{aligned}
A_{M, N, \Omega}(z, u) & =\frac{1}{2} \tau a_{M, N, \Omega}(z, u)+(z, u)_{\Omega}, \\
G_{M, N, \Omega}(z, u) & =-\frac{1}{2} \tau a_{M, N, \Omega}(z, u)+(z, u)_{\Omega} .
\end{aligned}
$$

Then, at each time step, we need to solve the equation

$$
\begin{aligned}
A_{M, N, \Omega}\left(u_{M, N}(t), \phi\right)= & G_{M, N, \Omega}\left(u_{M, N}(t-\tau), \phi\right) \\
& +\frac{1}{2} \tau(f(t)+f(t-\tau), \phi)_{\Omega}, \quad \forall \phi \in V_{M, N, \beta}^{0}(\Omega) .
\end{aligned}
$$

For notational convenience, let

$$
\begin{gathered}
\eta_{l}^{(\beta)}(x)=\tilde{\mathcal{L}}_{l}^{(\beta)}(x)-\tilde{\mathcal{L}}_{l+1}^{(\beta)}(x), \quad 0 \leq l \leq N-1 . \\
\zeta_{k}(y)=L_{k}(y)-L_{k+2}(y), \quad 0 \leq k \leq M-2 .
\end{gathered}
$$

In actual computation, we expand the numerical solution as

$$
u_{M, N}(x, y, t)=\sum_{l=0}^{N-1} \sum_{k=0}^{M-2} a_{k, l}(t) \eta_{l}^{(\beta)}(x) \zeta_{k}(y) .
$$

Let

$$
f_{k^{\prime} l^{\prime}}=\left(f(t), \eta_{l^{\prime}}^{(\beta)}(x) \zeta_{k^{\prime}}(y)\right)_{M, N, \beta, \Omega}, \quad 0 \leq k^{\prime} \leq M-2, \quad 0 \leq l^{\prime} \leq N-1,
$$

and define the vectors

$$
\begin{gathered}
X(t)=\left(a_{0,0}(t), a_{1,0}(t), \cdots, a_{M-2,0}(t), a_{0,1}(t), a_{1,1}(t), \cdots, a_{M-2,1}(t),\right. \\
\left.\cdots, a_{0, N}(t), a_{1, N}(t), \cdots, a_{M-2, N-1}(t)\right)^{T}, \\
F=\left(f_{0,0}(t), f_{1,0}(t), \cdots, f_{M-2,0}(t), f_{0,1}(t), f_{1,1}(t), \cdots, f_{M-2,1}(t),\right. \\
\left.\cdots, f_{0, N}(t), f_{1, N}(t), \cdots, f_{M-2, N-1}(t)\right)^{T} .
\end{gathered}
$$

Taking $\phi=\eta_{l^{\prime}}^{(\beta)}(x) \zeta_{k^{\prime}}(y)$ in (4.2) for $0 \leq k^{\prime} \leq M-2$ and $0 \leq l^{\prime} \leq N-1$, we find that (4.2) is equivalent to the following system of

$$
C_{11} X(t)=\widetilde{C}_{11} X(t-\tau)+\frac{1}{2} \tau\left(F_{1}(t)+F_{1}(t-\tau)\right)
$$

where

$$
\begin{aligned}
C_{11} & =A_{11} \otimes B_{11}+\frac{1}{2} \tau\left(a\left(A_{12} \otimes B_{12}\right)+b\left(A_{13} \otimes B_{13}\right)\right. \\
& \left.+\nu\left(A_{14} \otimes B_{14}\right)+\mu\left(A_{15} \otimes B_{15}\right)\right), \\
\widetilde{C}_{11} & =A_{11} \otimes B_{11}-\frac{1}{2} \tau\left(a\left(A_{12} \otimes B_{12}\right)+b\left(A_{13} \otimes B_{13}\right)\right. \\
& \left.+\nu\left(A_{14} \otimes B_{14}\right)+\mu\left(A_{15} \otimes B_{15}\right)\right),
\end{aligned}
$$


where the matrices

$$
A_{1 q}=\left(a_{l^{\prime} l}^{(1, q)}\right) \quad \text { and } \quad B_{1 q}=\left(b_{k^{\prime} k}^{(1, q)}\right), \quad 1 \leq q \leq 5
$$

with the entries

$$
\begin{aligned}
& a_{l^{\prime} l}^{(1,1)}=a_{l^{\prime} l}^{(1,3)}=a_{l^{\prime} l}^{(1,5)}=\int_{\Lambda} \eta_{l^{\prime}}^{(\beta)}(x) \eta_{l}^{(\beta)}(x) d x, a_{l^{\prime} l}^{(1,2)}=\int_{\Lambda} \eta_{l^{\prime}}^{(\beta)}(x) \partial_{x} \eta_{l}^{(\beta)}(x) d x, \\
& a_{l^{\prime} l}^{(1,4)}=\int_{\Lambda} \partial_{x} \eta_{l^{\prime}}^{(\beta)}(x) \partial_{x} \eta_{l}^{(\beta)}(x) d x, 0 \leq l^{\prime}, l \leq N-1,
\end{aligned}
$$

and

$$
\begin{aligned}
& b_{k^{\prime} k}^{(1,1)}=b_{k^{\prime} k}^{(1,2)}=b_{k^{\prime} k}^{(1,4)}= \begin{cases}\left(\zeta_{k^{\prime}}, \zeta_{k}\right)_{M, I}, & k^{\prime}=k=M-2, \\
\int_{I} \zeta_{k^{\prime}}(y) \zeta_{k}(y) d y, & \text { otherwise, }\end{cases} \\
& b_{k^{\prime} k}^{(1,3)}=\int_{I} \zeta_{k^{\prime}}(y) \partial_{y} \zeta_{k}(y) d y, \quad b_{k^{\prime} k}^{(1,5)}=\int_{I} \partial_{y} \zeta_{k^{\prime}}(y) \partial_{y} \zeta_{k}(y) d y, 0 \leq k, k^{\prime} \leq M-2 .
\end{aligned}
$$

We now calculate the entries of $A_{1 q}$ and $B_{1 q}, 1 \leq q \leq 5$. First, by (2.5) and (2.6) of [11], we obtain that for $0 \leq l^{\prime}, l \leq N$,

$$
\begin{aligned}
& a_{l^{\prime} l}^{(1,1)}=\left\{\begin{array}{ll}
-1 / \beta, & l^{\prime}=l-1, \\
2 / \beta, & l^{\prime}=l, \\
-1 / \beta, & l^{\prime}=l+1, \\
0, & \text { otherwise, }
\end{array} \quad a_{l^{\prime} l}^{(1,2)}= \begin{cases}-1 / 2, & l^{\prime}=l-1, \\
0, & l^{\prime}=l, \\
1 / 2, & l^{\prime}=l+1, \\
0, & \text { otherwise, }\end{cases} \right. \\
& a_{l^{\prime} l}^{(1,4)}= \begin{cases}\beta / 4, & l^{\prime}=l-1, \\
\beta / 2, & l^{\prime}=l, \\
\beta / 4, & l^{\prime}=l+1, \\
0, & \text { otherwise. }\end{cases}
\end{aligned}
$$

Next, we use (2.1) and (2.2) of [24] to obtain that for $0 \leq k^{\prime}, k \leq M-2$,

$$
\begin{aligned}
& b_{k^{\prime} k}^{(1,1)}=\left\{\begin{array}{ll}
\left(\zeta_{k^{\prime}}, \zeta_{k}\right)_{M, I}, & k^{\prime}=k=M-2, \\
-\frac{2}{2 k+1}, & k^{\prime}=k-2, \\
\frac{2}{2 k+1}+\frac{2}{2 k+5}, & k^{\prime}=k, \\
-\frac{2}{2 k^{\prime}+1}, & k^{\prime}=k+2, \\
0, & \text { otherwise, }
\end{array} \quad b_{k^{\prime} k}^{(1,3)}= \begin{cases}2, & k^{\prime}=k-1, \\
-2, & k^{\prime}=k+1, \\
0, & \text { otherwise, }\end{cases} \right. \\
& b_{k^{\prime} k}^{(1,5)}= \begin{cases}4 k+6, & k^{\prime}=k, \\
0, & \text { otherwise. }\end{cases}
\end{aligned}
$$

Now, we take the test function

$$
W(x, z, t)=\sin (x+y) / \sqrt{t+1} e^{-x^{2}} .
$$

Let $a=1, b=1, \nu=1$ and $\mu=1$ in (4.1). The numerical errors are measured by the discrete norm

$$
E_{M, N}(t)=\left\|W(t)-w_{M, N}(t)\right\|_{M, N, \beta, \Omega} .
$$




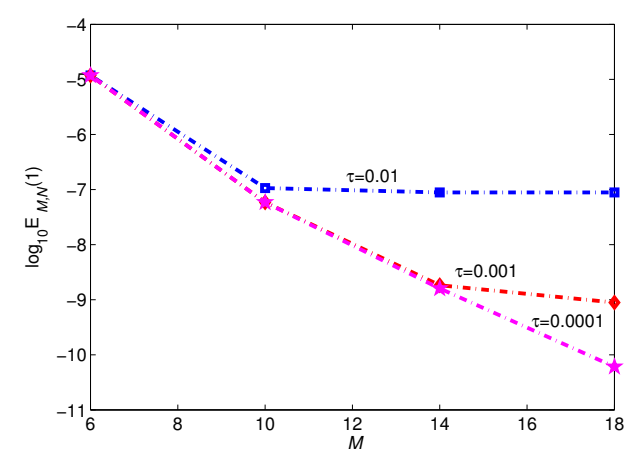

Figure 1. $\log _{10} E_{M, N}(1)$ vs $M$ of scheme (3.5) for (3.2) with different $\tau$.

In Figure 1, we plot the errors $\log _{10} E_{M, N}(t)$ with $N=5 M, t=1$ and $\beta=3$. Clearly, the errors decay fast when $M$ and $N$ increase and $\tau$ decreases. It is seen that for the fixed time step size $\tau=0.01$ and the small mode $M \leq 10$, the total numerical errors are dominated by the approximation errors in the space, and so they decay fast as $M$ increases. But for $M \geq 10$, the total numerical errors are dominated by the approximation errors in time $t$. Thus, the numerical solutions keep the same accuracy, even if $M$ and $N$ are further increased. A similar situation happens for $\tau=0.001$. On the other hand, for small $\tau \leq 0.0001$, the total numerical errors are dominated by the approximation errors in the space, and so they decay very fast as $M$ and $N$ increase. The above observations agree very well with theoretical analysis in Section 3.3. In particular, they show the spectral accuracy in the space of scheme (3.5).

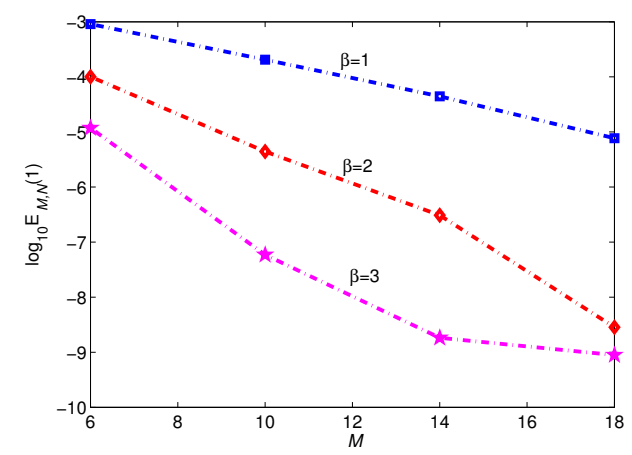

Figure 2. $\log _{10} E_{M, N}(1)$ vs $M$ of scheme (3.5) for (3.2) with different $\beta$.

In Figure 2, we plot $\log _{10} E_{M, N}(t)$ at $t=1$, with $N=5 M, \tau=0.001$ and different values of parameter $\beta$. It seems that the errors with suitably bigger $\beta$ are smaller than those with smaller $\beta$. However, how to choose the best 
parameter $\beta$ is still an open problem. Roughly speaking, if the exact solution decays faster as $x$ increases, then it is better to take suitably bigger $\beta$.

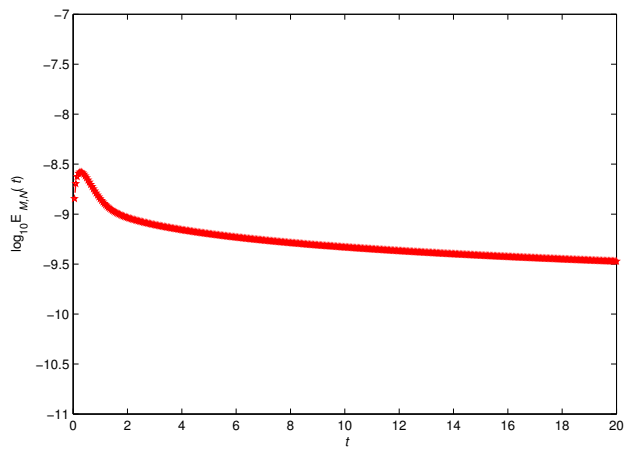

Figure 3. $\log _{10} E_{M, N}(t)$ vs $t$ of scheme (3.5) for (3.2).

In Figure 3, we plot the numerical errors $\log _{10} E_{M, N}(t)$ with $\beta=3, M=$ 25, $N=3 M, \tau=0.001$. They indicate the stability of scheme (3.5).

\section{Conclusions}

In this paper, we studied numerical simulation of non-isotropic heat transfer with Dirichlet boundary condition in an infinite strip. Since its solution decays exponentially as $x \rightarrow \infty$, it is better to use the Laguerre functions $e^{-\frac{1}{2} \beta x} \mathcal{L}_{l}^{(\beta)}(x)$ as the basis functions. Thus, we construct a mixed pseudospectral schemes which is the most appropriate for numerical simulation and save a lot of work. Moreover, the adjustable parameter $\beta$ offers great flexibility to match the asymptotic behaviors of exact solutions at infinity. The use of scaled generalized Laguerre functions leads to much simplified analysis, more precise error estimates. The numerical results indicate the spectral accuracy of the proposed scheme and coincide with theoretical analysis well.

In this paper, we treat the inhomogeneous Dirichlet data by means of a lifting so that the inhomogeneous boundary condition is satisfied exactly. In particular, the lifting in this paper is expressed explicitly and may also simulate the asymptotic behaviors of the solution of heat transfer. Moreover, we could combine the idea of [22] and this paper to design and analyze spectral methods for various mixed inhomogeneous boundary value problems defined on infinite strip. We shall report the related results in the future.

\section{Acknowledgments}

${ }^{a}$ This work of the first author is supported in part by NSF of China N.11371123, N.11571151, N.11171227, Fund of Henan Education Commission N.14B110021. ${ }^{b}$ This work of the second author is supported in part by NSF of China N. 11401380 and N.11326244. 
The authors thank sincerely the anonymous referees and Master Luyu Wang for their valuable suggestions and comments, which highly improve the quality of this article.

\section{References}

[1] Ch. Bernardi and Y. Maday. Spectral methods. In Techniques of Scientific Computing (Part 2), Handbook of Numerical Analysis, pp. 209-485. Elsevier, Amsterdam, 1997. http://dx.doi.org/10.1016/S1570-8659(97)80003-8.

[2] J.P. Boyd. Chebyshev and Fourier spectral methods. $2^{\text {nd }}$ edition. Dover Publication Inc., Mineola, New York, 2001.

[3] C. Canuto, M.Y. Hussaini, A. Quarteroni and Th.A. Zang. Spectral Methods. Springer-Verlag, Berlin, 2006.

[4] O. Coulaud, D. Funaro and O. Kavian. Laguerre spectral approximation of elliptic problems in exterior domains. Computer Methods in Applied Mechanics and Engineering, 80(1-3):451-458, 1990. http://dx.doi.org/10.1016/00457825(90)90050-V.

[5] D. Funaro. Polynomial Approximations of Differential Equations. SpringerVerlag, Berlin, 1992.

[6] D. Funaro and O. Kavian. Approximation of some diffusion evolution equations in unbounded domains by Hermite functions. Mathematics of Computation, 57(196):597-619, 1991. http://dx.doi.org/10.1090/S0025-5718-19911094949-X.

[7] B.-Y. Guo. Spectral methods and their applications. World Scientific, Singapore, 1998. http://dx.doi.org/10.1142/9789812816641.

[8] B.-Y. Guo and J. Shen. Laguerre-Galerkin method for nonlinear partial differential equations on a semi-infinite interval. Numerische Mathematik, 86(4):635654, 2000. http://dx.doi.org/10.1007/PL00005413.

[9] B.-Y. Guo, J. Shen and Ch.-L. Xu. Generalized Laguerre approximation and its applications to exterior problems. Journal of Computational Mathematics, 23(2):113-130, 2005.

[10] B.-Y. Guo and L.-L. Wang. Jacobi approximations in non-uniformly Jacobiweighted Sobolev spaces. Journal of Approximation Theory, 128(1):1-41, 2004. http://dx.doi.org/10.1016/j.jat.2004.03.008.

[11] B.-Y. Guo, L.-L. Wang and Z.-Q. Wang. Generalized Laguerre interpolation and pseudospectral method for unbounded domains. SIAM Journal on Numerical Analysis, 43(6):2567-2589, 2006. http://dx.doi.org/10.1137/04061324X.

[12] B.-Y. Guo and T.-J. Wang. Mixed Legendre-Hermite spectral method for heat transfer in an infinite plate. Computers $\&$ Mathematics with Applications, 51(5):751-768, 2006. http://dx.doi.org/10.1016/j.camwa.2006.03.004.

[13] B.-Y. Guo and T.-J. Wang. Composite Laguerre-Legendre spectral method for exterior problems. Advances in Computational Mathematics, 32(4):393-429, 2008. http://dx.doi.org/10.1007/s10444-008-9112-5.

[14] B.-Y. Guo and Ch.-L. Xu. Mixed Laguerre-Legendre pseudospectral method for incompressible fluid flow in an infinite strip. Mathematics of Computation, 73(245):95-125, 2004. http://dx.doi.org/10.1090/S0025-5718-03-01521-7. 
[15] B.-Y. Guo and K.-J. Zhang. On non-isotropic Jacobi pseudospectral method. Journal of Computational Mathematics, 26(4):511-535, 2008.

[16] B.-Y. Guo and X.-Y. Zhang. Spectral method for differential equations of degenerate type on unbounded domains by using generalized Laguerre functions. Applied Numerical Mathematics, 57(4):455-471, 2007. http://dx.doi.org/10.1016/j.apnum.2006.07.032.

[17] Y.-J. Jiao and B.-Y. Guo. Mixed spectral method for Navier-Stokes equations in an infinite strip by using generalized Laguerre functions. International Journal of Numerical Analysis and modeling, 9(4):982-998, 2012.

[18] Y. Maday, B. Pernaud-Thomas and H. Vandeven. Une tentative de rehabilitation des méthodes spéctrales de type Laguerre. Rech. Aérospat, 6:353-375, 1985.

[19] J. Shen. Stable and efficient spectral methods in unbounded domains using Laguerre functions. SIAM Journal on Numerical Analysis, 38(4):1113-1133, 2000. http://dx.doi.org/10.1137/S0036142999362936.

[20] J. Shen, T. Tang and L.-L. Wang. Spectral methods: Algorithms, Analysis and Applications. Springer Berlin Heidelberg, 2011. http://dx.doi.org/10.1007/9783-540-71041-7.

[21] T.-J. Wang. Generalized Laguerre spectral method for Fisher's equation on a semi-infinite interval. International Journal of Computer Mathematics, 92(5):1039-1052, 2015. http://dx.doi.org/10.1080/00207160.2014.920833.

[22] T.-J. Wang. Mixed spectral method for heat transfer with inhomogeneous Neumann boundary condition in an infinite strip. Applied Numerical Mathematics, 92:82-97, 2015. http://dx.doi.org/10.1016/j.apnum.2015.01.010.

[23] T.-J. Wang and B.-Y. Guo. Mixed Legendre-Hermite pseudospectral method for heat transfer in an infinite plate. Journal of Computational Mathematics, 23(6):587-602, 2005.

[24] T.-J. Wang and B.-Y. Guo. Composite Laguerre-Legendre pseudospectral method for exterior problems. Communications in Computational Physics, 5(24):350-375, 2009.

[25] T.-J. Wang and Y.-J. Jiao. Mixed generalized Laguerre-Legendre spectral method for heat transfer in an infinite strip. In Computational Sciences and $O p$ timization (CSO), 2012 Fifth International Joint Conference on, pp. 320-324. IEEE, 2012. http://dx.doi.org/10.1109/CSO.2012.78.

[26] Ch.-L. Xu and B.-Y. Guo. Laguerre pseudospectral method for nonlinear partial differential equations. Journal of Computational Mathematics, 20:413-428, 2002 .

[27] Ch.-L. Xu and B.-Y. Guo. Mixed Laguerre-Legendre spectral method for incompressible flow in an infinite strip. Advances in Computational Mathematics, 16(1):77-96, 2002. http://dx.doi.org/10.1023/A:1014249613222.

[28] X.-Y. Zhang and B.-Y. Guo. Spherical harmonic-generalized Laguerre spectral method for exterior problems. Journal of Scientific Computing, 27(1-3):523-537, 2006. http://dx.doi.org/10.1007/s10915-005-9056-6. 